\title{
ON UNIQUELY HAMILTONIAN CLAW-FREE AND TRIANGLE-FREE GRAPHS
}

\author{
Ben Seamone \\ Department d'Informatique et de recherche opérationnelle \\ Université de Montréal \\ Montreal, QC, Canada \\ e-mail: seamone@iro.umontreal.ca
}

\begin{abstract}
A graph is uniquely Hamiltonian if it contains exactly one Hamiltonian cycle. In this note, we prove that claw-free graphs with minimum degree at least 3 are not uniquely Hamiltonian. We also show that this is best possible by exhibiting uniquely Hamiltonian claw-free graphs with minimum degree 2 and arbitrary maximum degree. Finally, we show that a construction due to Entringer and Swart can be modified to construct triangle-free uniquely Hamiltonian graphs with minimum degree 3.
\end{abstract}

Keywords: Hamiltonian cycle, uniquely Hamiltonian graphs, claw-free graphs, triangle-free graphs.

2010 Mathematics Subject Classification: 05C38, 05C45.

\section{REFERENCES}

[1] S. Abbasi and A. Jamshed, A degree constraint for uniquely Hamiltonian graphs, Graphs Combin. 22 (2006) 433-442. doi:10.1007/s00373-006-0666-z

[2] H. Bielak, Chromatic properties of Hamiltonian graphs, Discrete Math. 307 (2007) $1245-1254$.

doi:10.1016/j.disc.2005.11.061

[3] J.A. Bondy and B. Jackson, Vertices of small degree in uniquely Hamiltonian graphs, J. Combin. Theory (B) 74 (1998) 265-275. doi:10.1006/jctb.1998.1845

[4] R.C. Entringer and H. Swart, Spanning cycles of nearly cubic graphs, J. Combin. Theory (B) 29 (1980) 303-309. doi:10.1016/0095-8956(80)90087-8 
[5] H. Fleischner, Uniquely Hamiltonian graphs of minimum degree 4, J. Graph Theory 75 (2014) 167-177.

doi: $10.1002 /$ jgt. 2172

[6] P. Haxell, B. Seamone and J. Verstraete, Independent dominating sets and Hamiltonian cycles, J. Graph Theory 54 (2007) 233-244. doi:10.1002/jgt.20205

[7] J. Petersen, Die theorie der regulären graphs, Acta Math. 15 (1891) 193-220. doi:10.1007/BF02392606

[8] J. Sheehan, The multiplicity of Hamiltonian circuits in a graph, in: Recent Advances in Graph Theory (Proc. Second Czechoslovak Sympos., Prague, 1974), Fiedler $($ Ed(s)), (Prague: Academia, 1975) 477-480.

[9] A.G. Thomason, Hamiltonian cycles and uniquely edge colourable graphs, Ann. Discrete Math. 3 (1978) 259-268.

doi:10.1016/S0167-5060(08)70511-9

[10] C. Thomassen, On the number of Hamiltonian cycles in bipartite graphs, Combin. Probab. Comput. 5 (1996) 437-442.

doi:10.1017/S0963548300002182

[11] C. Thomassen, Independent dominating sets and a second Hamiltonian cycle in regular graphs, J. Combin. Theory (B) 72 (1998) 104-109. doi10.1006/jctb.1997.1794

[12] W.T. Tutte, On Hamiltonian circuits, J. London Math. Soc. 21 (1946) 98-101. doi:10.1112/jlms/s1-21.2.98

Received 20 January 2014

Revised 2 April 2014

Accepted 2 April 2014 